\title{
Treatment of refractory post-esophagectomy anastomotic esophageal strictures using temporary fully covered esophageal metal stenting compared to repeated bougie dilation: results of a randomized controlled trial
}

\section{다 (1) $(-)$}

\author{
Authors \\ E. van Halsema², P. D. Siersema ${ }^{1,5}$ \\ Institutions \\ 1 University Medical Center Utrecht, Utrecht, The \\ Netherlands \\ 2 Academic Medical Center, Amsterdam, The Netherlands \\ 3 Erasmus University Medical Center, Rotterdam, The \\ Netherlands \\ 4 Instituto do Cancer do Estado de Sao Paulo, Sao Paulo, \\ Brazil \\ 5 Radboud University Medical Center, Nijmegen, The \\ Netherlands
}

W. F. Kappelle', J. E. van Hooft ${ }^{2}$, M. C. W. Spaander ${ }^{3}$, F. P. Vleggaar ${ }^{1}$, M. J. Bruno ${ }^{3}$, F. Maluf-Filho ${ }^{4}$, A. Bogte ${ }^{1}$,

submitted 26.4.2018

accepted after revision 24.9.2018

\author{
Bibliography \\ DOI https://doi.org/10.1055/a-0777-1856 | \\ Endoscopy International Open 2019; 07: E178-E185 \\ (c) Georg Thieme Verlag KG Stuttgart · New York \\ ISSN 2364-3722
}

Corresponding author

Peter D. Siersema, MD, PhD, Dept. of Gastroenterology and Hepatology, Radboud University Medical Center, Nijmegen, The Netherlands

Fax: +31 104658520

peter.siersema@radboudumc.nl

\section{ABSTRACT}

Background and study aims Fully covered self-expanding metal stents (FCSEMS) provide an alternative to bougie dilation (BD) for refractory benign esophageal strictures. Controlled studies comparing temporary placement of FCSES to repeated $B D$ are not available.

Patients and methods Patients with refractory anastomotic esophageal strictures, dysphagia scores $\geq 2$, and two to five prior BD were randomized to 8 weeks of FCSEMS or to repeated $\mathrm{BD}$. The primary endpoint was the number of BD during the 12 months after baseline treatment.

Results Eighteen patients were included (male $67 \%$, median age 66.5; 9 received metal stents, 9 received BD). Technical success rate of stent placement and stent removal was $100 \%$. Recurrent dysphagia occurred in 13 patients ( $72 \%$ ) during follow-up. No significant difference was found between the stent and BD groups for mean number of BD during follow-up (5.4 vs. 2.4, $P=0.159$ ), time to recurrent dysphagia (median 36 days vs. 33 days, Kaplan-Meier: $P=$ 0.576 ) and frequency of reinterventions per month (median 0.3 vs. $0.2, P=0.283$ ). Improvement in quality of life score was greater in the stent group compared to the BD group at month 12 (median $26 \%$ vs. $4 \%, P=0.011$ ).

Conclusions The current data did not provide evidence for a statistically significant difference between the two groups in the number of BD during the 12 months after initial treatment. Metal stenting offers greater improvement in quality of life from baseline at 12 months compared to repeated BD for patients with refractory anastomotic esophageal strictures.

\section{Introduction}

Benign esophageal strictures are a challenging clinical problem and may occur secondary to gastroesophageal reflux disease, external beam radiation, caustic ingestion, or surgical anastomosis following esophagectomy [1,2]. A common cause of benign strictures of the esophagus is development of anastomotic strictures following resection of the distal esophagus and proximal stomach for treatment of esophageal cancer $[3,4]$. The mainstay of management of benign esophageal strictures is esophageal dilation. Several techniques for dilation exist and the most commonly used ones are Savary-Gilliard bougie dilation (BD) and through-the-scope (TTS) balloon dilation [5]. 
While $80 \%$ to $90 \%$ of patients initially respond to dilation, most patients require more than one session to reach adequate dilation, and nearly $10 \%$ will experience a refractory or recurrent stricture despite repeated dilation [6]. Perforation is the most serious complication of esophageal dilation, and carries a mortality rate of approximately $20 \%$ [7]. The overall perforation rate has been reported to be $0.1 \%$ to $0.4 \%$, but rates as high as $1.9 \%$ have been reported with BD [8]. The rate of significant bleeding after dilation, likely the result of mucosal disruption caused by dilation, has been reported to be $0.4 \%$. Furthermore, retrosternal pain is reported in $0 \%$ to $5 \%$ of cases after dilation [9].

Adjuncts to management of difficult benign esophageal strictures include steroid injection, cautery, and topical application of the antiproliferative agent mitomycin C. Most recently, esophageal stenting techniques have been used in patients with esophageal strictures. Options for stenting include nonmetal (plastic) expandable stents, biodegradable stents, and self-expanding metal stents (SEMS) [6,10-15]. Over the past 20 years, SEMS have become available in a wide variety of materials and configurations. Stainless steel and nitinol (an alloy of nickel and titanium) are the most common materials used in SEMS design, and the wire mesh construction of the stent can be braided or woven [16]. SEMS are also available in uncovered (bare) versions as well as versions that are partially covered (PC) or fully covered (FC) with silicone membranes. PC and FCSEMS are designed to reduce tissue ingrowth through the mesh of the stent. As a result, the FCSEMS appear to allow for longterm removability, thus permitting their use in both malignant and benign disease $[10,12,17]$.

Use of FCSEMS for management of postoperative complications following esophagectomy has not been well studied. However, several studies have included small numbers of patients with refractory anastomotic esophageal strictures who were treated with FCSEMS [18-21]. Results from these studies have indicated that while FCSEMS are in place, generally over a period of a few months, the patients experience a reduction in dysphagia symptoms associated with anastomotic strictures, but symptoms return shortly after the stent is removed in all but $20 \%$ to $30 \%$ of patients. In addition, these studies report frequent complications associated with esophageal stenting, particularly stent migration and erosion of tissue surrounding the stent. Our study adds to the literature a randomized comparison of anastomotic esophageal stricture treatment using temporary placement of a FCSEMS to repeated BD.

\section{Patients and methods}

\section{Study design}

This was a prospective, multicenter, randomized, controlled, open-label clinical trial. The study was conducted in the Netherlands and Brazil and was approved by the ethics committee of the University Medical Center Utrecht, the Netherlands, and thereafter by the ethics committees of all participating centers. In Brazil, the study was also approved by the Brazilian regulatory Competent Authority prior to enrollment. All patients provided written informed consent at the time of enrollment.
The study was conducted in accordance with the latest version of the Declaration of Helsinki. The trial was sponsored by Boston Scientific Corporation and is registered at www.ClinicalTrials.gov, number NCT01699542. All procedures were performed by endoscopists with extensive experience with treatment of esophageal diseases, including benign esophageal strictures.

\section{Patients}

Patients with a refractory symptomatic esophagogastric anastomotic stricture post-esophagectomy were enrolled at four centers, three in the Netherlands and one in Brazil. Eligible patients were 18 years or older and had esophageal anastomotic benign stricture post-esophagectomy (esophagogastric strictures), which was performed at the same institution where patient enrollment and follow-up was planned; baseline Ogilvie dysphagia scores of Grade 2 (ability to swallow semi-solid foods), Grade 3 (ability to swallow liquids only), or Grade 4 (unable to swallow liquids) [22]; between two and five prior dilations to at least $16 \mathrm{~mm}$ in diameter (first dilation no more than 6 months and last dilation no more than 24 months post-esophagectomy); a stricture unable to pass with a standard endoscope of approximately $9.8 \mathrm{~mm}$ diameter, and were willing and able to comply with study procedures and provide written informed consents. Major exclusion criteria included strictures within $2 \mathrm{~cm}$ of the upper esophageal sphincter, strictures more than $5 \mathrm{~cm}$ in length, dysphagia related to a motility disorder, non-anastomotic esophageal strictures, esophagocolonic strictures, prior esophageal stent placement, and active erosive esophagitis.

\section{Procedures}

Patients were randomized 1:1 to endoscopic BD or temporary placement of a FCSEMS, the WallFlex Esophageal FC stent (Boston Scientific, Marlboro, Massachusetts, United States) which is made of nitinol wires braided to form a cylindrical mesh. The stent contains a flare at both ends to minimize risk of migration. A suture is threaded just on the proximal end and may be used to remove or reposition the stent. Stent sizes were 18-mm body diameter and 103-, 123-, or 153-mm length; or 23-mm body diameter with 105-, 125-, or 155-mm length.

The WallFlex stent was chosen for this study because it is an easy-to-place stent, even in inexperienced hands. Because it is available in a fully-covered version, it is amenable for use for benign indications. The stent has a good safety record with a low risk of adverse events (AEs). Finally, the combination of radial and axial force and the flexibility of the stent theoretically reduce the risk of stent migration [23].

Patients in the SEMS group were treated with a single FCSEMS at time 0 , with endoscopic imaging confirmation after placement. FCSEMS were removed per protocol at 8 weeks $( \pm 7$ days) post-placement or sooner if complications occurred, at the discretion of the treating physician and patient. After stent removal, dilation for recurrence of dysphagia was repeated as needed, and these dilation procedures were recorded until end of study at 12 months after enrollment. The BD group received dilations up to at least $16 \mathrm{~mm}$, in one to four dilation ses- 
sions, per standard treatment; the date of the last dilation session was considered time 0 , completion of the initial study treatment. In cases of recurrent dysphagia in the BD group, dilation was repeated and recorded until 12 months after enrollment.

\section{Follow-up}

After initial study treatment, all patients completed an in-person or by-phone visit on Day 2, Week 2, and Months 1, 2, 3, 6, 9 , and 12 . In the SEMS group, the Month 2 visit coincided with endoscopic removal of the FCSEMS. At each follow-up visit, information on AEs was collected, Quality of life (QoL) was evaluated per the EQ-5D-5L questionnaire, a validated questionnaire for describing and valuing general health based on five dimensions including mobility, self-care, usual activities, pain/ discomfort, and anxiety/depression [24]. A patient's reported pain and satisfaction with therapy was assessed using a 10point visual analog scale (VAS) and episodes of dysphagia (at baseline, daily for the first month and weekly thereafter up to 12 months) were evaluated as recorded in the patient's diary, and endoscopic and/or fluoroscopic imaging was conducted in the FCSEMS group at the discretion of the investigator. Each subject was followed for 12 months post-initial study treatment.

\section{Endpoints}

The primary endpoint was the number of dilation procedures for management of dysphagia within 12 months following initial study treatment. Secondary endpoints included AEs related to the device and/or procedure; technical stent placement success, defined as the ability to deploy the stent in satisfactory position across the stricture with a standard 9.-mm endoscope; technical stent removal success, defined as the ability to remove the stent without complications; time to recurrence of dysphagia, defined as inability to pass the stricture; stent migration with or without symptoms; number of reinterventions within 12 months following the initial study treatment; dysphagia scores throughout follow-up; patient report of pain and satisfaction with the therapy (by VAS); and change in QoL.

\section{Sample size calculation}

Based on prior experience of the senior investigator (PS), after initial treatment with a SEMS, approximately half the patients do not require dilation procedures for management of dysphagia, and approximately half require one to three dilations in the subsequent 12 months [2]. After initial treatment with BD, approximately $30 \%$ of patients need at most one dilation and approximately $70 \%$ of patients need two to four dilations in the 12-month period [5]. This yields an expected number of dilations of 1.0 with standard deviation (SD) of 1.15 in the SEMS group, and 2.3 with SD of 1.39 in the BD group. The hypothesis of the study was superiority of the mean number of dilation procedures in the SEMS group compared to the BD group, with a margin of $1.30,80 \%$ power and two-sided alpha of 0.05 . Testing this hypothesis required 20 patients per group. Adding $10 \%$ of patients to compensate for potential attrition during followup yielded the initial 44-patient enrollment goal. Due to slow enrollment, the study was discontinued after 33 months when 18 patients had been enrolled and completed the 12-month follow-up.

\section{Randomization}

Patients were randomized to a 1:1 ratio between the Stent Arm (Group A) and BD Arm (Group B). Randomization was stratified by clinical site using random block sizes of four. Randomization sequence was created using SAS version 9.3 and executed at the time of initial treatment by study staff at each participating center. After a patient was enrolled and passed screening, site staff referred to the randomization sequence to determine treatment assignment. An electronic database was the primary method to implement randomization with envelopes as a backup method. At the end of trial enrollment, a total of 18 patients were randomized, with nine assigned to the Stent Group and nine to the BD Group.

\section{Statistical analyses}

Baseline data (patient demographics and medical history) and post-procedure information was summarized using descriptive statistics (e.g., mean, standard deviation, number of data points used) for continuous variables with normal distribution or count variables, interquartile range for continuous variable with non-normal distribution, and frequency tables for discrete variables. A negative binomial model was used to compare the primary endpoint and the number of dilation procedures for management of dysphagia during 12-month follow-up between the BD and SEMS group. Univariate and multivariate analysis were performed per protocol to assess possible predictors of the primary endpoint. Factors from the univariate model with $P \leq 0.20$ were modeled multivariately using a stepwise procedure in a negative binomial model. The significance thresholds for entry and exit into the model was set to $P<0.10$. Change in overall QoL health score was analyzed using repeated measures analysis.

\section{Results}

\section{Participant flow}

A total of 22 patients were assessed for eligibility, of whom four were excluded for not meeting inclusion criteria (3 patients) and investigative site error during enrollment ( 1 patient). Eighteen patients were deemed eligible and were subsequently randomized, as shown in $>$ Fig. 1.

\section{Baseline}

Baseline (BL) characteristics were similar between the two study groups ( $\triangleright$ Table 1 ). Mean age was $67 \pm 7$ years and $67 \%$ of patients were male. Esophagectomy was transthoracic in $78 \%(14 / 18)$ and transhiatal in $22 \%(4 / 18)$. Mean time from esophagectomy to first dilation was $95 \pm 36$ days. Mean stricture length was $0.7 \mathrm{~cm}$ (range $0.3-1.5 \mathrm{~cm}$ ), located at $21.6 \pm$ $2.9 \mathrm{~cm}$ from the incisors. The SEMS group had lower baseline QoL scores (median 65 vs. 80, $P=0.021$ ), but similar dysphagia scores (median 2 vs. 2, $P=0.206$ ) and number of BDs up to 12 months prior to baseline treatment (mean 2 vs. $2.4, P=0.566$ ). 


\section{Enrollment}

\section{Assessed for eligibility $(n=22)$}

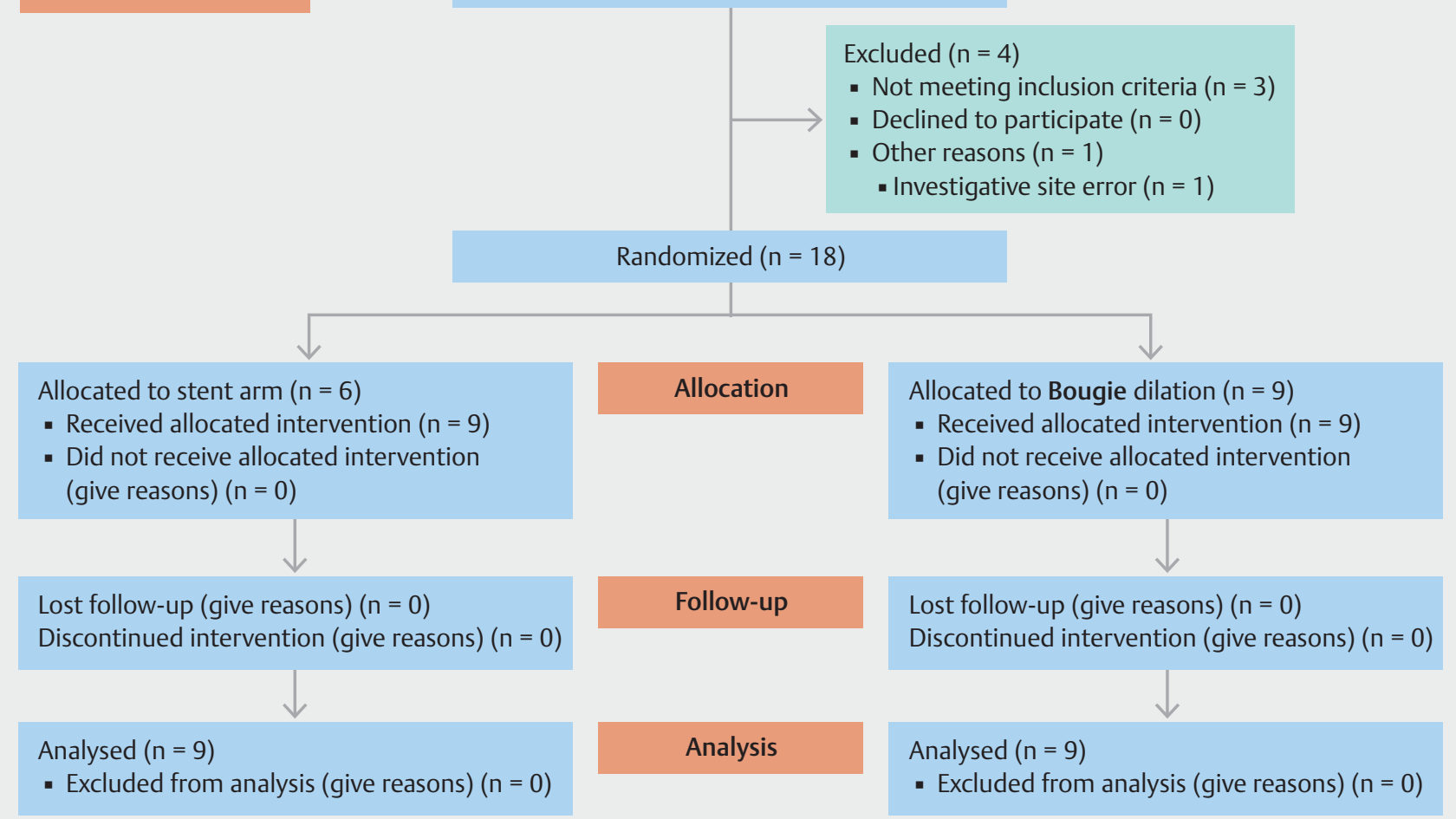

- Fig. 1 CONSORT diagram of study flow.

- Table 1 Demographics and baseline information.

\begin{tabular}{|c|c|c|c|}
\hline & BD & SEMS & P value ${ }^{1}$ \\
\hline Age & $66.6 \pm 7.7$ & $66.6 \pm 6.3$ & 1.0 \\
\hline Male & $66.7 \%(6 / 9)$ & $66.7 \%(6 / 9)$ & 1.0 \\
\hline Type of esophagectomy & & & 0.577 \\
\hline Transthoracic & $88.9 \%(8 / 9)$ & $66.7 \%(6 / 9)$ & \\
\hline Transhiatal & $11.1 \%(1 / 9)$ & $33.3 \%(3 / 9)$ & \\
\hline Max dilation (second dilation) (mm) & $15.9 \pm 2.0(9)$ & $16.4 \pm 0.9(9)$ & 0.450 \\
\hline Dysphagia score ${ }^{2}$ & $2.0(2.0,2.0)$ & $2.0(2.0,3.0)$ & 0.206 \\
\hline Dysphagia score $=2$ & $100 \%(9 / 9)$ & $66.7 \%(6 / 9)$ & 0.206 \\
\hline Dysphagia score $>2$ & $0.0 \%(0 / 9)$ & $33.3 \%(3 / 9)$ & 0.206 \\
\hline Number of pretreatment dilations & $2.4 \pm 1.0$ & $2.0 \pm 0.0$ & 0.566 \\
\hline Quality of life overall health score & $80.0(70.0,80.0)$ & $65.0(50.0,70.0)$ & 0.021 \\
\hline
\end{tabular}

BD, bougie dilation; SEMS, self-expanding metal stents. Treatment group is per randomization.

${ }^{1} P$ values calculated from $t$-test for continuous variable with normal distribution, Wilcoxon test for continuous variable with non-normal distribution, negative

binomial models for count variable, and Fisher's exact for binary variable.

${ }^{2}$ Median (IQR) presented for continuous variables with non-normal distribution. 
- Table 2 Post-initial treatment dilations and reinterventions.

\begin{tabular}{|c|c|c|c|}
\hline & BD & SEMS & $P$ value $^{1}$ \\
\hline Number of post-dilations due to dysphagia through 12 months & $2.4 \pm 2.5$ & $5.4 \pm 5.4$ & 0.159 \\
\hline Number of post-dilations through 12 months & $2.7 \pm 2.6$ & $5.4 \pm 5.4$ & 0.183 \\
\hline Frequency of dilation per month post-treatment ${ }^{2,3}$ & $0.2(0.1,0.3)$ & $0.3(0.2,1.1)$ & 0.283 \\
\hline Total number of reinterventions & $2.9 \pm 2.7$ & $5.6 \pm 5.3$ & 0.168 \\
\hline Time to first recurrence of dysphagia ${ }^{3}$ & $33(21,33)$ & $36(24,71)$ & 0.576 \\
\hline Reintervention due to dysphagia & $88.5 \%(23 / 26)$ & $98.0 \%(49 / 50)$ & 0.113 \\
\hline \multicolumn{4}{|l|}{ Reason for dysphagia recurrence } \\
\hline Stent migration & $0.0 \%(0 / 23)$ & $6.1 \%(2 / 33)$ & 0.507 \\
\hline New stricture formation & $4.3 \%(1 / 23)$ & $0.0 \%(0 / 33)$ & 0.411 \\
\hline Stricture recurrence & $95.7 \%(22 / 23)$ & $87.9 \%(29 / 33)$ & 0.639 \\
\hline Other & $0.0 \%(0 / 23)$ & $6.1 \%(2 / 33)^{4}$ & 0.507 \\
\hline \multicolumn{4}{|l|}{ Type of Reintervention } \\
\hline Study stent removed & $0.0 \%(0 / 26)$ & $4.0 \%(2 / 50)$ & 0.544 \\
\hline Bougie dilation & $96.2 \%(24 / 26)$ & $94.0 \%(47 / 50)$ & 1.0 \\
\hline Balloon dilation & $0.0 \%(0 / 26)$ & $4.0 \%(2 / 50)$ & 0.544 \\
\hline Other & $7.7 \%(2 / 26)$ & $20.0 \%(10 / 50)^{5}$ & 0.202 \\
\hline \multicolumn{4}{|c|}{$\begin{array}{l}\text { BD, bougie dilation; SEMS, self-expanding metal stents } \\
\text { Treatment group is per randomization. } \\
\text { KM estimated median (IQR) presented for time to event variables. } \\
{ }^{1} P \text { values calculated from Wilcoxon test for continuous variable with non-normal distribution, negative binomial models for count variable, fisher's exact for binary } \\
\text { variable, and log-rank test for time to event variable. } \\
{ }^{2} \text { Defined as time } 0 \text { to last follow-up or end of study for BD and stent removal to last follow-up or end of study for SEMS } \\
{ }^{3} \text { Median (IQR) presented for continuous variables with non-normal distribution and } \\
{ }^{4} \text { One patient experienced two incidences of recurrent dysphagia; cause unknown } \\
{ }^{5} \text { Other types of reintervention are: gastroscopy to check for recurrent stenosis (1) and gastroscopy performed but no stenosis (1) for BD and needle knife (9) and } \\
\text { Chest tube into right pleura and pleurodesis (1) for SEMS. }\end{array}$} \\
\hline
\end{tabular}

\section{Initial treatment}

Nine FCSEMS were placed, seven patients received an $18-\mathrm{mm}$ FCSEMS with a length of $103 \mathrm{~mm}$ and two patients received a 23-mm FCSEMS with a length of $105 \mathrm{~mm}$. Technical placement and removal success in the SEMS group was $100 \%$. SEMS were removed by retrieval of the suture at the proximal end of the SEMS in $67 \%$ of patients (6/9) and from the stomach in $33 \%$ of patients (3/9). Two patients required early removal due to symptomatic stent migration.

Among the nine BD patients, six had a single dilation for initial treatment $(16 \mathrm{~mm}$ for 3 patients, $17 \mathrm{~mm}$ for 1 patient and $18 \mathrm{~mm}$ for 2 patients), two patients had two dilation sessions (14 mm followed by $16 \mathrm{~mm}$ after 15 days in 1 patient and 15 $\mathrm{mm}$ followed by $17 \mathrm{~mm}$ after 16 days in 1 patient), and one patient had three dilation sessions $(14 \mathrm{~mm}$ followed by $16 \mathrm{~mm}$ after 7 days and $18 \mathrm{~mm}$ after 8 days).

\section{Treatment of recurrent dysphagia}

Post-treatment dilations and reinterventions are listed in $\mathbf{D a} \mathbf{T a}$ ble 2. Dysphagia was the cause of reintervention in $89 \%(23 / 26)$ of the BD group and $98 \%$ (49/50) of the SEMS group. In the BD group, other causes for reintervention are as followings: one patient had a gastroscopy to check for recurrent stenosis, one patient had odynophagia and a third patient reported complaints with passing food but a slight stricture could be passed with a diagnostic scope (approx. 9.8-mm diameter) and hence this was not qualified as dysphagia caused by the stricture. In the SEMS group, one patient had a reintervention due to pleuritic carcinomatosa of the right lung and had a chest tube into the right pleura and pleurodesis. The mean number of posttreatment dilations for dysphagia was not different between the groups (2.4 for the BD group; 5.4 for the SEMS group; $P=$ $0.159)$. A Kaplan-Meier analysis of time to first recurrence after initial treatment for the BD group and after removal for the SEMS group demonstrated no difference between groups ( $P=$ 0.576 ) ( $\triangleright$ Fig. 2). Median time to recurrence of dysphagia after treatment was 33 days for the BD group and 36 days for the SEMS group. The most common reason for recurrent dysphagia was stricture recurrence. The most common reintervention was $\mathrm{BD}, 92 \%$ of the interventions in the BD group and $94 \%$ in the SEMS group. Both groups experienced similar reductions in frequency of dilation (calculated as number of dilations divided by duration from first to last dilation) after the initial study treatment $(P=0.931)$. In the BD group, median frequency of dilation 


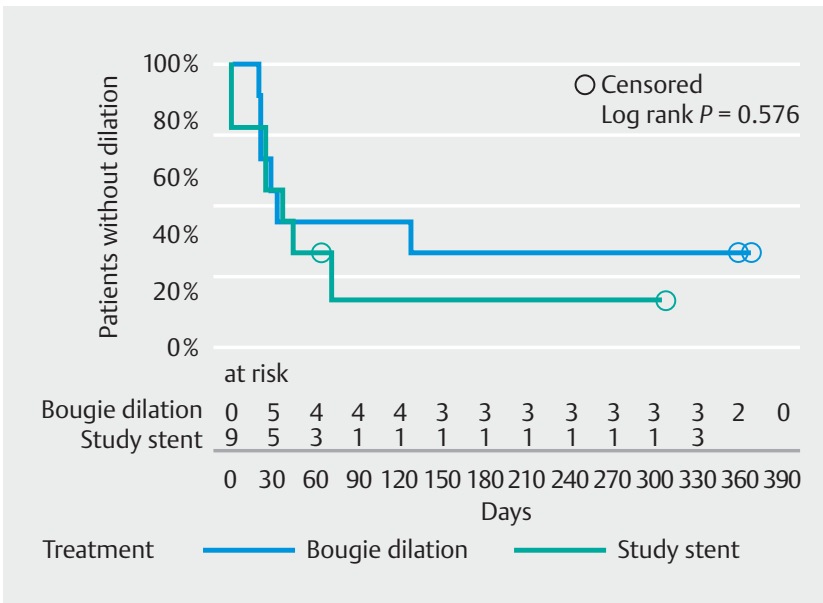

- Fig. 2 Kaplan-Meier analysis of time to first recurrence. The start time of the 12-month follow-up period for the bougie dilation (BD) group was the same as time 0 , which is defined as the end of the initial treatment period. However, for the stent (SEMS) group, the start time of follow-up was the time of stent removal rather than stent placement.

- Table 3 Multivariate analysis of number of reinterventions due to dysphagia.

\begin{tabular}{|l|l|l|}
\hline Predictor & $\begin{array}{l}\text { Rate ratio } \\
\mathbf{9 5} \mathbf{C l}\end{array}$ & Pvalue $^{\mathbf{1}}$ \\
\hline SEMS vs. BD & $\begin{array}{l}2.4 \\
(1.0,5.7)\end{array}$ & 0.053 \\
\hline Location of anastomotic stricture & $\begin{array}{l}0.7 \\
(0.5,0.9)\end{array}$ & 0.006 \\
\hline
\end{tabular}

$\mathrm{BD}$, bougie dilation; SEMS, self-expanding metal stent

${ }^{1}$ Negative binomial model is used.

decreased from 2.7 before study treatment to 0.2 interventions per month after study treatment. In the SEMS group, the median frequency decreased from 2.9 to 0.3 .

\section{Multivariate analysis}

A multivariate analysis identified location of anastomotic stricture, age and treatment modality - SEMS or BD - as predictors of the number of reinterventions due to dysphagia ( $\triangleright$ Table 3 ). Age was not a significant predictor at the 0.05 level when adjusting for other covariates. Multivariate analysis demonstrated that with each centimeter increase of distance from the incisors to the stricture, the number of reinterventions for dysphagia during 12 months after the initial treatment decreased by $32 \%$ $(P=0.006)$.

\section{Patient-reported outcomes}

Pain by VAS in the SEMS group was generally low, with median ranging from 0 at baseline to a maximum of 3 at Day 2, and a return to low levels (median 0 ) at Day 14 which remained low during follow-up. In the BD group, pain levels were consistently low (median 0 ) during follow-up. Patient satisfaction with treat-

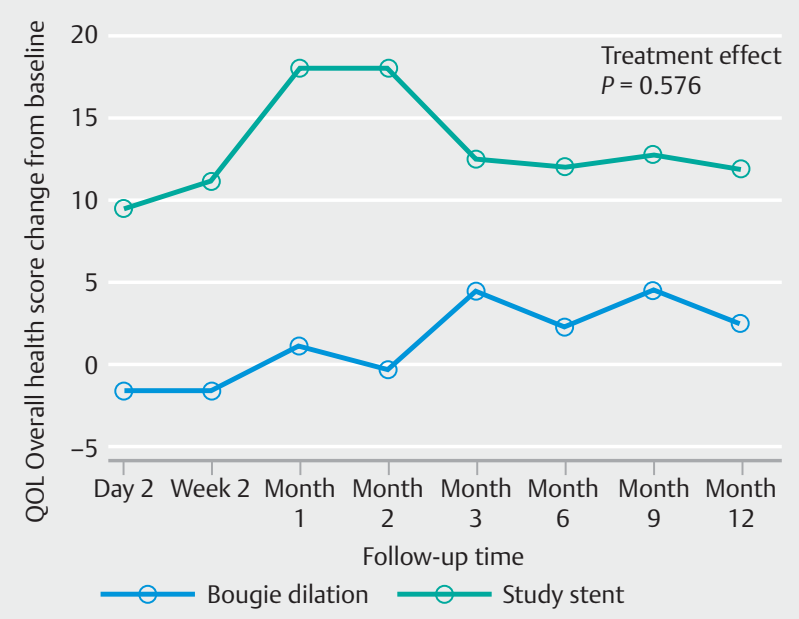

- Fig. 3 Change from baseline in quality of life (QoL) scores using the EQ-5D-5L questionnaire. The repeated measures analysis showed a significant effect of treatment on overall health score change ( $P=0.043)$ as well as a significant effect of time $(P=0.028)$. No significant interaction effect between treatment and time was found.

ment was similar between the groups (SEMS median ranging from 8 to 9.5; BD median ranging from 8 to 10). The repeated measures analysis on QoL overall health score change suggests a significant difference between SEMS group and the BD group $(P=0.043)$. As seen in $>$ Fig. 3 , patients in the SEMS group seemed to experience a greater QoL improvement following initial study treatment compared with the BD group. It is possible that the significant difference in baseline scores between the two groups may have contributed to the greater improvement in the SEMS group. In other words, the SEMS group improved more because there was more room for improvement in this group. Additionally, when interpreting the QoL improvement, one should consider the under-enrollment and the small sample size and the similar satisfaction scores between the two groups. It is possible that patients were equally satisfied with the treatment but SEMS patients experienced higher improvement in overall QoL due to fewer procedures to manage dysphagia while the stent was in place. Details of patient-reported outcomes, including VAS pain score and patient satisfaction score, are shown in $>$ Fig. 4 and $>$ Fig. $\mathbf{5}$.

\section{Adverse events}

A total of eight AEss were related to either the procedure or the device, of which seven were non-serious and one serious. In the SEMS group, one patient experienced aspiration during FCSEMS removal (serious), one experienced recurrent dysphagia due to stent migration, one had stent-related gastroesophageal reflux, one had foreign body sensation and retching due to foreign body sensation (both related to stent), one experienced thoracic pain and cervical pain during FCSEMS placement procedure, and one patient had epigastric pain after FCSEMS removal. 


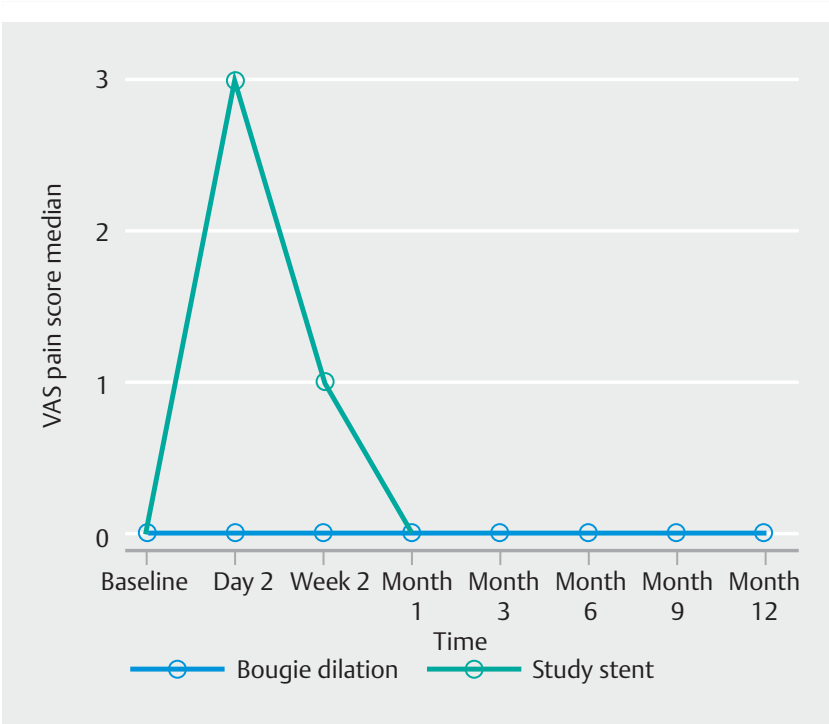

Fig. 4 Visual analog scale (VAS) pain scores.

\section{Discussion}

The current study confirmed that FCSEMS placement is a technically feasible and a clinically effective treatment for benign refractory anastomotic strictures after esophagectomy during FCSEMS indwell, including greater QoL improvement during FCSEMS treatment compared with continued BD. However, the number of dilations required in the 12 months after treatment were comparable between the two groups. Patients' reports of pain and satisfaction were similar between the groups.

The study failed to confirm our initial hypothesis, namely that placement of a FCSEMS would reduce the number of dilations over a follow-up time of 12 months after initial treatment. Several factors may have contributed to this outcome. First, the sample size was smaller than intended, thus complicating detection of small differences between the two methods. Based on the 18 subjects randomized, the power to detect the predefined relevant difference in the primary outcome was low and consequently the chance of detecting a true effect may be reduced. Similarly, multivariate analysis results and QoL results should be interpreted with caution. Due to slow enrollment over 33 months, it was decided to discontinue enrollment before the predefined required number of patients was included. The main reason for slow enrollment is that patients tend to be more willing to receive standard of care than to try out a new treatment, therefore, one inclusion criterion was relaxed, namely the number of dilations to reach $16 \mathrm{~mm}$ in diameter since esophagectomy. Originally this was two dilations, but was expanded to allowing two to five dilations. Another limitation was that time 0 started at the last dilation session for the BD group and at stent placement for the SEMS group, because that time point reflects the time at which the stricture is first dilated to the largest diameter. That, however, led to the patients in the SEMS group having a 2-month shorter window to document potential recurrence of dysphagia during 12 months of follow-up after time 0 . To adjust for this issue, the Kaplan

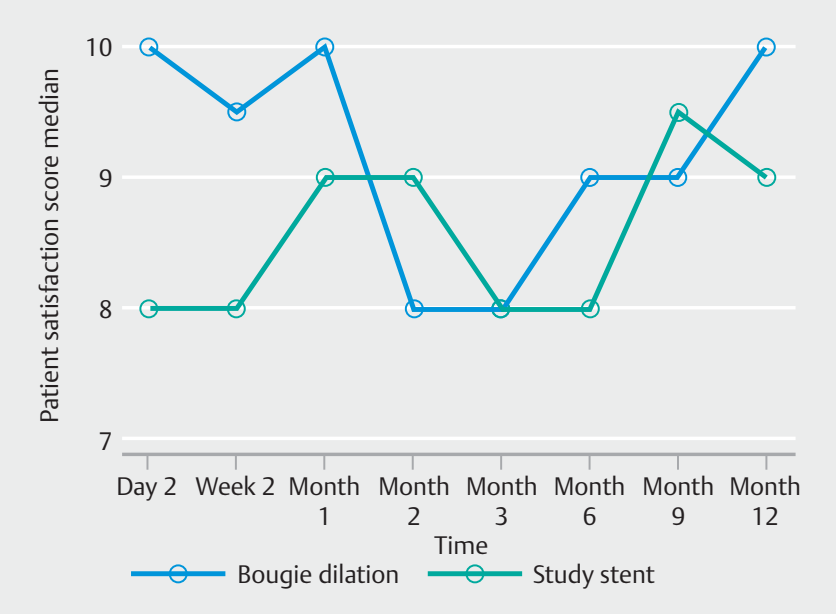

Fig. 5 Patient satisfaction with treatment.

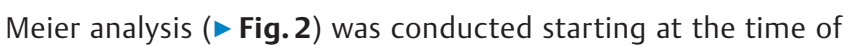
stent removal for the SEMS group and at the time of the last dilation for the BD group.

Other limitations of the study include lack of data on details on the anastomotic technique and that the caliber of stricture was not evaluated by barium study. Gastroesophageal reflux disease (GERD) is a common occurrence in esophagectomy patients and may contribute to esophageal pathology. In future studies, the potential role of possible associated GERD should be considered.

In addition, there were no clear criteria for dilation at the time of recurrence of dysphagia in the study. Some patients received reintervention to manage dysphagia without an observed increase in dysphagia score.

In the current study, the stent was removed after 8 weeks to avoid tissue overgrowth with new stricture formation as a result. During the time that the stent was in place, dilations for dysphagia were avoided; after removal of the stent, the median number of episodes of dysphagia requiring intervention increased. These findings suggest that stent placement for benign strictures is effective, but only as long as the stent is in place. Its effect is not sustained once the stent has been removed. Some anastomotic strictures are highly refractory due to ongoing ischemia leading to recurrent fibrosis.

The number of related AEs was higher in the SEMS group. Based on literature and our own experience, we see no reason to suspect a direct causal relationship in the finding of increased number of related events, and instead believe this to be an artifact of the small sample size.

Despite not meeting the primary endpoint, patients in the SEMS group experienced a higher QoL improvement from baseline compared with the BD group, and the differences between two groups were significant at Months 1 and 2 as well as toward the end of the follow-up (at Months 9 and 12). Note that QoL score at baseline in the SEMS group was significantly lower than in the BD group. To account for baseline scores, QoL score change from baseline in percentage was calculated instead of the raw score. The peak improvement of the QoL in the SEMS 
group occurred at the time of stent removal (8 weeks). Both groups had decreased frequency of interventions for dysphagia during the study. Larger studies are needed to evaluate the recurrent dysphagia rate for FCSEMS vs. standard dilation in patients with refractory anastomotic strictures. Longer-term utilization of FCSEMS and outcome measures comparing interventions during the time of stenting only versus a standard dilation group would be relevant outcome measures for subsequent studies.

\section{Conclusion}

It can be concluded that in the current study, no significant difference was found between SEMS placement and repeat BD with regard to the number of BDs thereafter. Nevertheless, temporary FCSEMS indwell may offer reduced treatment burden as long as the stent remains in place and offers similar (if not greater) QoL improvement from baseline sustained for 12 months compared to repeat BD for patients with refractory anastomotic esophageal strictures. In addition, biodegradable stents could be considered as a patient-friendly alternative for FCSEMS placement, as stent removal is avoided.

\section{Acknowledgements}

The authors acknowledge Pooja G. Goswamy, Zehua Liu, Matthew Rousseau, and Joyce Peetermans from Boston Scientific Corporation for contributions to study design, study conduct, data analysis, and critical review of the manuscript.

\section{Competing interests}

\section{None}

\section{References}

[1] Eloubeidi MA, Lopes TL. Novel removable internally fully covered selfexpandable metal esophageal stent: feasibility, technique of removal and tissue response in humans. Am J Gastroenterol 2009; 104: 1374 1381

[2] van Boeckel PG, Siersema PD. Refractory strictures: what to do when dilation fails. Curr Treat Option Gastroenterol 2015; 13: 47-58

[3] Conio M, Blanchi S, Filiberti R et al. Self-expanding stents in benign esophageal strictures. Tech Gastrointest Endosc 2008; 10: 164-174

[4] Repici A, Hassan C, Sharma P et al. Systemic review: the role of selfexpanding plastic stents for benign oesophageal strictures. Aliment Pharmacol Ther 2010; 31: 1268-1275

[5] Grooteman KV, Wong Kee Song LM, Vleggaar FP et al. Non-adherence to the rule of 3 does not increase the risk of adverse events in esophageal dilation. Gastrointest Endosc 2017; 85: 332 - 337
[6] Sigounas DE, Siddhi S, Plevris JN. Biodegradable esophageal stents in benign and malignant strictures - a single center experience. Endosc Int Open 2016; 4: E618-E623

[7] Brinster C], Singhal S, Lee L et al. Evolving options in the management of esophageal perforation. Ann Thorac Surg 2004; 77: 1475-1483

[8] Fry LC, Monkemuller K, Neumann $\mathrm{H}$ et al. Incidence, clinical management and outcomes of esophageal perforations after endoscopic dilatation. Z Gastroenterol 2007; 45: 1180 -1184

[9] Lew RJ, Kochman ML. A review of endoscopic methods of esophageal dilation. J Clin Gastroenterol 2002; 35: 117-126

[10] Ferguson DD. Evaluation and management if benign esophageal strictures. Dis Esophagus 2005; 18: 359 - 364

[11] Bakken JC, Song WK, de Groen PC et al. Use of a fully covered self-expandable metal stent for the treatment of benign esophageal diseases. Gastrointest Endosc 2010; 72: 712 - 720

[12] Lee JG, Hsu R, Leung JW. Are self-expanding metal mesh stents usefu in the treatment of benign esophageal stenoses and fistulas? An experience of four cases Am J Gastroenterol 2000; 95: 1920-1925

[13] Hirdes MM, van Hooft JE, Wijrdeman HK et al. Combination of biodegradable stent placement and single-dose brachytherapy is associated with an unacceptably high complication rate in the treatment of dysphagia from esophageal cancer. Gastrointest Endosc 2012; 76: $267-274$

[14] van Halsema EE, Wong Kee Song LM, Baron TH et al. Safety of endoscopic removal of self-expandable stents after treatment of benign esophageal diseases. Gastrointest Endosc 2013; 77: 18-28

[15] Spaander MC, Baron TH, Siersema PD et al. Esophageal stenting for benign and malignant disease: European Society of Gastrointestinal Endoscopy (ESGE) Clinical Guideline. Endoscopy 2016; 48: 939 - 948

[16] Schembre D. Advances in esophageal stenting: the evolution of fully covered stents for malignant and benign disease. Adv Ther 2010; 27 : $413-425$

[17] van Boeckel PG, Vleggaar FP, Siersema PD. Biodegradable stent placement in the esophagus. Expert Rev Med Devices 2013; 10: 37 43

[18] Eloubedi MA, Talreja JP, Lopes TL et al. Success and complications associated with placement of fully covered removable self-expandable metal stents for benign esophageal diseases (with videos). Gastrointest Endosc 2011; 73: 673-681

[19] Liu J, Hu Y, Cui C et al. Removable, fully covered, self-expandable metal stents for the treatment of refractory benign esophagogastric anastomotic strictures. Dysphagia 2012; 27: 260 - 264

[20] Speer E, Dunst CM, Shada A et al. Covered stents in cervical anastomoses following esophagectomy. Surg Endosc 2016; 30: 3297 - 3303

[21] Repici A, Small AJ, Mendelson A et al. Natural history and management of refractory benign esophageal strictures. Gastrointest Endosc 2016; 84: $222-228$

[22] Ogilvie AL, Dronfield MW, Ferguson R et al. Palliative intubation of oesophagogastric neoplasms at fibreoptic endoscopy. Gut 1982; 23 : $1060-1067$

[23] Hirdes MMC, Siersema PD, Vleggar FP. A new fully covered metal stent for the treatment of benign and malignant dysphagia: a prospective follow-up study. Gastrointest Endosc 2012; 75: 712-718

[24] The EuroQol Group. EuroQol-a new facility for the measurement of health-related quality of life. Health Policy 1990; 16: 199-208 